\title{
AVALIAÇÃO DA CAPACIDADE FUNCIONAL DOS IDOSOS DURANTE 0 PERÍODO DE ISOLAMENTO SOCIAL PELO COVID-19
}

Natália Guedes Alves; Universidade do Estado do Pará (UEPA); natyguedes31@gmail.com

Allana Moura de Araújo; Universidade do Estado do Pará (UEPA); allanauepa@gmail.com

Ana Luísa Gross Rodrigues; Universidade do Estado do Pará (UEPA); analuisagross11@gmail.com

Beatriz Vieira Leite Rodrigues; Universidade do Estado do Pará (UEPA); biavlrodrigues2@gmail.com Carlos Vinicius Carrera do Nascimento; Universidade do Estado do Pará (UEPA); viniciuscarrera@gmail.com Cynthia Cyllene de Oliveira Charone; Grupo Cynthia Charone (GCC); cynthiacharone@yahoo.com.br Rondinei Silva Lima; Grupo Cynthia Charone (GCC); nep@hospitalcynthiacharone.com Fabíola de Souza Abrahão; Grupo Cynthia Charone (GCC); nep@hospitalcynthiacharone.com

\section{RESUMO}

Introdução: Segundo a Classificação Internacional de Funcionalidade, Incapacidade e Saúde, da OMS, funcionalidade é a interação dinâmica entre a condição de saúde de uma pessoa, os fatores ambientais e fatores pessoais. No cenário do isolamento social, essas variáveis podem ser afetadas, modificando a funcionalidade de idosos. Deste modo, estudos sobre como a funcionalidade de idosos pode ser afetada durante a pandemia do COVID-19, auxiliam na segurança das condutas para essa população. Objetivo: Avaliar a capacidade funcional dos idosos assistidos no serviço de saúde do GCC, durante o isolamento social pelo COVID-19. Métodos: Estudo transversal e descritivo, com amostra de 799 pacientes, avaliados mediante telemonitoramento. Foram incluídas pessoas com 60 anos ou mais, participantes do serviço de geriatria e gerontologia do Grupo Cynthia Charone e que aceitaram participar voluntariamente, mediante assinatura do estudo e aprovação do CEP. Resultados: Dos pacientes analisados, 40,7\% estavam na faixa etária de 60 a 69 anos. Cerca de 84,1\% apresentaram de 1-4 comorbidades, destacando-se HAS (60,2\%), DM $(24,8 \%)$ e OA (19\%). 23,5\% precisaram de ajuda para realizar atividades, $81 \%$ apresentou dificuldade de levantar da cadeira e 27,4\% apresentou problemas de memória. Conclusão: No cenário da pandemia, doenças prévias incluem o paciente em um grupo de maior risco de desfechos desfavoráveis, assim, é importante prevenir o contágio nesta faixa etária, para reduzir possíveis incapacidades causadas pelo COVID-19. É preciso acompanhar pacientes que já possuem algum tipo de incapacidade, para atrasar a progressão de doenças crônicas.

Palavras-chave : Idoso; Isolamento social; Infecções por coronavírus. 